\title{
COMPARING THE KINEMATICS OF BACK SQUATS PERFORMED WITH DIFFERENT HEEL ELEVATIONS
}

original paper

() Wroclaw University of Health and Sport Sciences

DOI: https://doi.org/10.5114/hm.2021.106164

\section{PEDRO HENRIQUE MARTINS MONTEIRO ${ }^{1}$, ALEXANDRE JEHAN MARCORI ${ }^{1}$, VITOR BERTOLI NASCIMENTO ${ }^{2}$, ANDERSON NASCIMENTO GUIMARÃES ${ }^{2}$, VICTOR HUGO ALVES OKAZAKI ${ }^{2}$}

\author{
${ }^{1}$ University of São Paulo, São Paulo, Brazil \\ ${ }^{2}$ State University of Londrina, Londrina, Brazil
}

\begin{abstract}
Purpose. Back squat (BS) is a popular exercise owing to its capacity to develop lower limb strength. During BS, trunk inclination and knee range of motion (RoM) are relevant aspects of a proper technique, and these movement kinematics parameters can be positively altered with official weightlifting shoes lifting the heel $13 \mathrm{~mm}$ above the ground. Wedges are a low-cost alternative to lifting the heel to different heights, but movement kinematics adaptations with higher elevations, above $25 \mathrm{~mm}$, are not well described in the literature. Thus, we compared the effect of different heights of heel wedges on BS kinematics. Methods. Fifteen experienced recreational weightlifters (22 \pm 5.4 years; $83 \pm 11 \mathrm{~kg}$; $179 \pm 6 \mathrm{~cm}$; $5 \pm 2.1$ years of BS experience) were conveniently selected. Three randomized conditions were applied: barefoot (B), 25-mm (W25), and 50-mm wedges (W50). BS movement was assessed by kinematic analysis with an optoelectronic camera system.
\end{abstract}

Results. After ANOVA, the post-hoc indicated significant RoM differences in reducing trunk $(F=27.27 ; p<0.01)$ and increasing knee $(F=16.87 ; p<0.01)$ flexions between conditions. Post-hoc analysis verified decreasing trunk inclination $(\mathrm{B}>\mathrm{W} 25>\mathrm{W} 50 ; p<0.05)$ and increasing knee $(\mathrm{B}<\mathrm{W} 25<\mathrm{W} 50 ; p<0.05)$ RoM with increasing wedge height.

Conclusions. Higher wedges allowed positive adaptations by promoting a more upright trunk position and greater BS depth. Using a heel wedge can be a low-cost and viable strategy to optimize BS technique in a variety of training settings and contexts. Key words: resistance training, range of motion, injury prevention, biomechanics, wedge

\section{Introduction}

Back squat is a major exercise used in resistance training [1] and several sports modalities. An organized and systematic practice of back squat may promote lower limb strength and power gains [2], hypertrophy [3], assistance in rehabilitation processes [4], and maintenance of elderly strength and functionality [5]. Given the enhanced applicability of this exercise, performing it properly is essential for different goals and sports. Within this context, range of motion (RoM) is an aspect of performance that needs to be cautiously considered during back squats. Besides being proportionally related to different gluteus maximus activation [6] during back squat, higher RoMs on key joints (e.g. knee) also promote better adaptations in resist- ance training programs mainly owing to increased time under tension: a significant variable that aids muscle gains [7]. Recent experimental evidence showed that performing a full squat is superior to half squat training regarding gluteus maximus and adductor hypertrophy [3]. Another issue that deserves attention in back squat kinematics is the lower limb and lumbar spine joints overload. For instance, these areas are related to increased injury risk during a back squat, especially when performing with inappropriate form or heavy loads [8-10]. This increases muscle torque and shear forces on the lumbar spine, which interact better with compressive forces that shear [1]. Therefore, proper movement technique is crucial.

Previous research has shown that adequate technique is related to the distance between feet, appropri-

Correspondence address: Pedro Henrique Martins Monteiro, University of São Paulo, Av. Professor Mello Moraes, 65 - Vila Universitaria, São Paulo - SP, 05508-030, Brazil, e-mail: pedrohm96@hotmail.com

Received: September 21, 2020

Accepted for publication: December 4, 2020

Citation: Monteiro PHM, Marcori AJ, Nascimento VB, Guimarães AN, Okazaki VHA. Comparing the kinematics of back squats performed with different heel elevations. Hum Mov. 2022;23(2):97-103; doi: https://doi.org/10.5114/hm.2021.106164. 
ated shoes wearing, and vertical trunk position (to mention a few) [1], since these points decrease shear forces at lower limbs and lumbar spine [1]. To improve some of these aspects, it has been documented that using proper weightlifting shoes while back squatting can increase knee RoM [11]. Weightlifting shoes are designed with a 13-mm elevation between the forefoot and the heel; this parameter has been defined by the International Weightlifting Federation and the value is sufficient to promote a more vertical trunk position, greater RoM in the knee joint, and better stability during back squat $[12,13]$. While these investigations suggest technical positive adaptations with weightlifting shoes, more recent research has been unable to verify benefits of squatting with raised heel, since similar kinematics were observed when participants squatted barefoot [14, 15]. Additionally, general practitioners include other training methods on regular routines, like post-activation potential, being more practical with using running shoes than weightlifting shoes. Hence, an alternative approach to lifting the heel during this exercise can be an adapted wedge without a standard height, such as iron plates or a squared wooden block. With this approach, it would be possible to evaluate the effects of squatting with the heel elevated above the 13-mm height provided by weightlifting shoes. For instance, Charlton et al. [16] described positive outcomes (e.g. less trunk flexion) with a $25-\mathrm{mm}$ wooden block beneath the heel during back squat in trained male subjects. In turn, Lee et al. [14] did not verify modifications in the knee, thoracic, or lumbar joint kinematics when comparing back squat performed barefoot vs. with 33-mm elevated heels. It is important to highlight that Lee et al. [14] investigated recreational weightlifters, while Charlton et al. [16] assessed trained participants. Considering that most skilled participants present a gold standard technique [17], investigating this population is a way to attribute kinematic differences by using wedges.

In the previous evidence, the literature remains inconclusive regarding the benefits of squatting with the heel elevated above $25 \mathrm{~mm}$, and no investigation up to date has analysed the modifications of back squat kinematics using wedges higher than those. Thus, to understand the greater height that promotes positive adaptations, without an uncomfortable heel elevation, can be useful for practitioners to apply wedges in the height range in daily practice. Furthermore, frontal plane analysis of the hip joint (adduction and abduction movements) is lacking in the previous research. Given this scenario, this topic still needs further research to clarify whether lifting the heel while back squatting is an adequate strategy to optimize biomechanical movement parameters. This study aimed to describe the modifications that heel wedges can promote in back squat kinematics. We analysed the kinematics of the lower limb joints (ankle, knee, and hip) and trunk motion in 3 back squat conditions: barefoot (B), 25-mm wedge (W25), and 50-mm wedge (W50). We set $50 \mathrm{~mm}$ as this was the height limit to lift the heel with comfort. Considering previous evidence of load influence in knee and hip kinematics during back squat [18], and that load may promote fatigue (which is a potentially confounding effect in our results), it was decided that the back squat would be implemented without load in this study. Thus, our study aimed to assess maximal RoM without external interferences and with minimal variation of technique across trials. We hypothesized that a higher heel elevation would (H1) decrease anterior trunk inclination and (H2) increase knee and ankle RoM. The results can contribute to better understanding the influence of an elevated heel during back squat kinematics, aiding an adequate prescription that can be considered in different contexts by coaches, athletes, resistance training professionals, and practitioners.

\section{Material and methods}

\section{Participants}

The sample size was calculated by G*Power 3.1 (Franz Faul, Germany) for a repeated-measures ANOVA within factors ( 1 group $\times 3$ measures), considering a moderate effect size according to Cohen $(d=0.5)$, $\alpha$ error probability $=0.05$, and power $(1-\beta)=0.8$, following the recommendations by Faul et al. [19]. On the basis of these parameters, a minimum of 9 subjects was estimated.

A total of 15 men, resistance-trained adults $(22 \pm$ 5.4 years of age, $83 \pm 11 \mathrm{~kg}, 179 \pm 6 \mathrm{~cm}$ ), were conveniently selected for this study. They had been injuryfree in the lower limbs and trunk for at least 6 months before the research. All subjects had a minimum experience with back squat exercise of 3 years $(5 \pm 2.1$ years on average), performing it at least once a week in their training routine.

\section{Procedures}

The participants performed the exercise shirtless, barefoot, and with standardized shorts. We positioned a wooden block below the participants' heel (on calcaneus) to provide the elevations during both experi- 
A

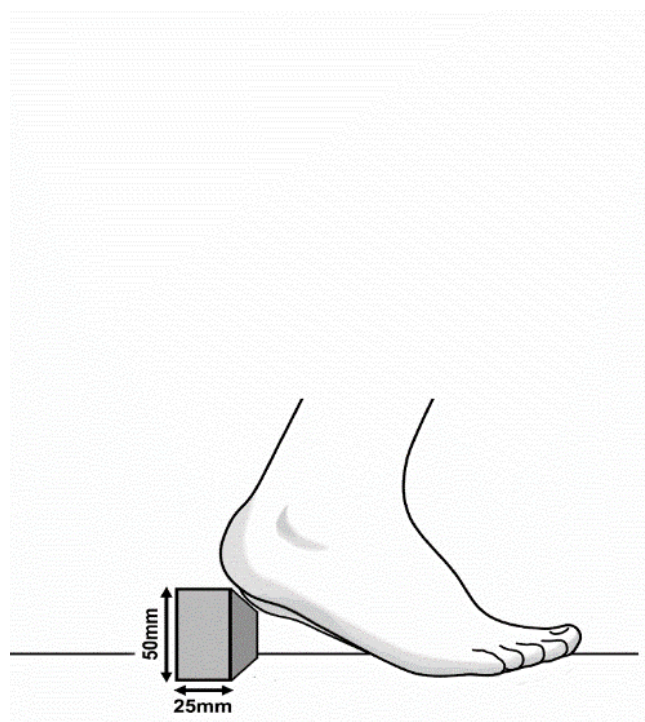

B

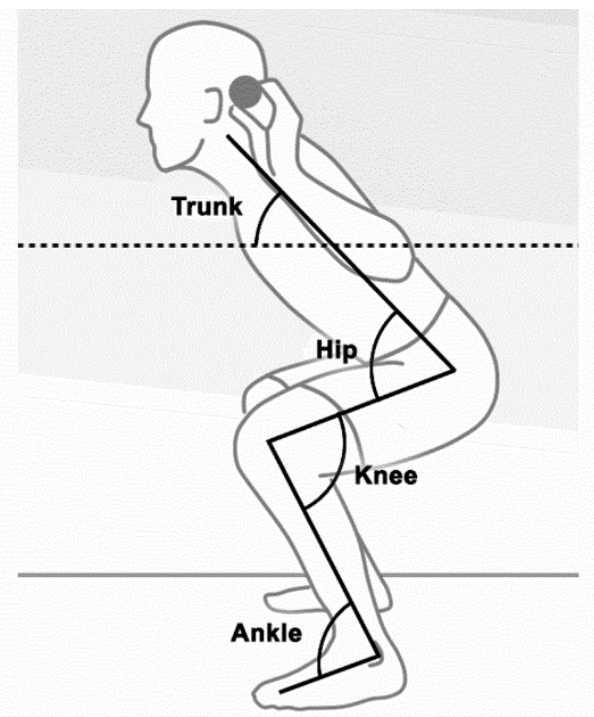

Figure 1. Wooden block representation (A) and sagittal plane angle interpretations (B) mental conditions (W25 and W50), as represented in Figure 1A. For this study, we made 2 wooden blocks that presented the same measures to support calcaneus between the different heights. For kinematic analyses, reflective markers were positioned on the subject's skin, and the camera manufacturer's calibration procedure was followed. Next, a warm-up set (10 repetitions) in each experimental condition (B, W25, and W50) was performed, followed by rate of perceived exertion scale answer to ensure that the participants were not fatigued. This procedure was in line with the literature recommendations of Borg scale, and all subjects had previous experience with the scale. On resting 2 minutes after the warm-up, the participants performed 3 sets of 6 repetitions in each experimental condition (B, W25, and W50), and rate of perceived exertion scale was measured after each series. The subjects were instructed to perform the back squat with full RoM in all conditions. Another 2-minute sitting interval between sets was allowed. A light wooden stick (Figure 1B) was used to replace the iron bar during the back squat to simulate movement kinematics without additional overload. The condition orders were randomized among the subjects, and a researcher was responsible for replacing the wedge when necessary. Movement speed was controlled by a digital metronome ( 2 seconds eccentric, 1 second concentric), and the first warm-up series served as a familiarization protocol for the metronome tempo. A black dot fixed at the eye level was positioned on the wall (2 $\mathrm{m}$ away) to standardize the visual focus.
Motion analysis

Movement kinematics were assessed by 7 optoelectronic cameras (MX-T-Series) of the Vicon System (v. 1.8.5, Vicon Motion System, Oxford, UK) with a sampling frequency of $100 \mathrm{~Hz}$. The cameras were positioned in a way that at least 2 of them visualized the reflective markers throughout the movement, avoiding gaps during data collection and ensuring accuracy. A total of 35 reflective markers were positioned in the subject's body, in accordance with the Vicon Plug-in Gait Full Body model. The following variables were analysed: maximum, minimum, and RoM of relative angles of hip flexion and abduction, knee flexion, and dorsiflexion, as well as maximum, minimum, and RoM of absolute angles of trunk inclination. Angles in the sagittal plane were interpreted as presented in Figure 1B. The hip abduction angle was interpreted with $180^{\circ}$ in the anatomic position, with decreasing angles during abduction and increasing angles during adduction. The right body side was analysed.

All variables (minimum, maximum, and RoM) were acquired in each repetition, and then averaged for each set. Finally, the average of the 3 sets in each condition was used for statistical purposes. Repetition beginning was determined when the spatial position of the C7 marker reached a reduction greater than $4 \mathrm{~mm}$ from one frame to another (eccentric phase), while repetition ending was determined when the same marker reached the highest spatial value (concentric phase). The deepest point of the squat movement was determined on the basis of the minimum value of the knee flexion angle. 


\section{HUMAN MOVEMENT}

P. Monteiro et al., Effect of different wedges on back squat kinematics

\section{Data processing}

Data were processed by using Vicon Nexus (v. 1.8.5, Vicon Motion system, Oxford, UK). The time series of the joint angles were filtered with a low-pass recursive digital Butterworth filter $\left(4^{\text {th }}\right.$ order, a cut-off frequency of $3 \mathrm{~Hz}$ ). The filtering parameters were determined after spectral and residual analysis of the signal [20]. Filtering and analysis, as well as the calculation of the variables, were executed by a personalized MATLAB routine (2017a, MathWorks, USA).

\section{Statistical analyses}

Normality and sphericity were verified by ShapiroWilk's and Mauchly's test, respectively. Mean and standard deviation were used to describe the angles and joint RoMs, while repeated-measures ANOVA compared the RoM between the different heel elevations in each joint. Bonferroni's post-hoc test was performed when necessary. Effect sizes were calculated for RoM in a paired measure design (B vs. W50), considering the correlation between both measures [21], and interpreted in accordance with Cohen [22] in light of recommendations by Rhea [23] for trained subjects. All analyses were conducted with the SPSS software (v. 21.0, IBM Statistics), and significance was set at $p<0.05$.

\section{Ethical approval}

The research related to human use has complied with all the relevant national regulations and institu- tional policies, has followed the tenets of the Declaration of Helsinki, and has been approved by the State University of Londrina Ethics Committee.

\section{Informed consent}

Informed consent has been obtained from all individuals included in this study.

\section{Results}

The results showed significant differences in the trunk, knee, and ankle RoM between conditions (Table 1). Post-hoc comparisons verified decreased RoM in the absolute trunk inclination with increasing wedge height ( $\mathrm{B}>\mathrm{W} 25>\mathrm{W} 50 ; p<0.05)$. RoM in knee flexion increased as the wedges were higher $(\mathrm{B}<\mathrm{W} 25<$ W50; $p<0.05$ ), while for dorsiflexion, RoM was only significantly increased between the W25 and the W50 conditions $(p<0.05)$. When comparing B against W50, effect sizes indicated a strong effect for absolute trunk $(d=1.97)$, knee $(d=1.23)$, and ankle $(d=4.68)$ RoM. No significant differences were verified in the results of hip flexion or adduction.

\section{Discussion}

The heel elevation height significantly affected back squat kinematics, decreasing anterior trunk inclination and increasing knee and ankle RoM. Hence, both hypotheses initially raised ( $\mathrm{H} 1$ and $\mathrm{H} 2)$ were accepted. As such, elevating the heel at 25 and $50 \mathrm{~mm}$ can produce positive adaptations in back squat kinematics,

Table 1. Range of motion, minimum and maximum displacement of joints during back squat with different heel elevations

\begin{tabular}{|c|c|c|c|c|c|}
\hline & B & W25 & W50 & & \\
\hline Parameter & $\begin{array}{l}\text { RoM / Min-Max } \\
\text { (SD) }\end{array}$ & $\begin{array}{l}\text { RoM / Min-Max } \\
\text { (SD) }\end{array}$ & $\begin{array}{l}\text { RoM / Min-Max } \\
\text { (SD) }\end{array}$ & $F$ & $p$ \\
\hline Trunk $\left(^{\circ}\right)$ & $\begin{array}{c}32.8 / 176.1-143.2 \\
(7.1 / 5.9-7.8)\end{array}$ & $\begin{array}{c}28.6 / 176.8-148.1 \\
(7.9 / 6.0-7.8)\end{array}$ & $\begin{array}{c}25.3 / 177.0-151.8^{\mathrm{a}, \mathrm{b}} \\
(7.5 / 5.9-7.4)\end{array}$ & 27.27 & $<0.001$ \\
\hline Hip flexion $\left({ }^{\circ}\right)$ & $\begin{array}{c}98.6 / 170.4-71.8 \\
(13.6 / 6.5-10.7) \\
\end{array}$ & $\begin{array}{l}97.5 / 170.0-72.5 \\
(13.1 / 7.0-10.11) \\
\end{array}$ & $\begin{array}{c}95.9 / 170.1-74.1 \\
(11.9 / 6.8-9.6)\end{array}$ & 2.67 & 0.107 \\
\hline Hip adduction $\left(^{\circ}\right)$ & $\begin{array}{c}19.2 / 172.9-153.7 \\
(7.5 / 3.2-7.7)\end{array}$ & $\begin{array}{c}19.9 / 173.3-153.4 \\
(6.3 / 2.7-6.9)\end{array}$ & $\begin{array}{c}19.3 / 172.7-153.4 \\
(5.6 / 2.9-6.3)\end{array}$ & 1.28 & 0.165 \\
\hline Knee $\left(^{\circ}\right)$ & $\begin{array}{c}115.1 / 175.7-60.6 \\
(17.8 / 5.3-15.9)\end{array}$ & $\begin{array}{c}121.7 / 174.3-52.6 \\
(15.8 / 6.8-12.5)\end{array}$ & $\begin{array}{c}126.2 / 173.6-47.4^{\mathrm{a}, \mathrm{b}} \\
(11.6 / 7.6-8.0)\end{array}$ & 16.87 & $<0.001$ \\
\hline Ankle $\left(^{\circ}\right)$ & $\begin{array}{c}32.3 / 93.1-60.7 \\
(6.5 / 3.6-4.8)\end{array}$ & $\begin{array}{c}36.7 / 97.9-61.2 \\
(11.4 / 4.0-9.9)\end{array}$ & $\begin{array}{c}38.2 / 103.0-64.8^{\mathrm{b}} \\
(11.6 / 4.0-10.4)\end{array}$ & 6.97 & 0.009 \\
\hline
\end{tabular}

B - barefoot, W25 - 25-mm wedge, W50 - 50-mm wedge, RoM - range of motion

${ }^{\mathrm{a}} p<0.05$ vs. $\mathrm{B},{ }^{\mathrm{b}} p<0.05$ vs. W25 
being possibly applied in different training programs with various exercising goals. Our results corroborate previous studies in the literature, allowing to further understand the effect of elevating the heel during back squat execution and providing information on how professionals and practitioners can use it in exercise programs that involve back squatting.

Lumbar joints are among the most injury-prone areas during back squatting, excessive anterior trunk inclination seems to be the leading cause $[8,10]$. Therefore, our results reveal that elevating the heel at either 25 or $50 \mathrm{~mm}$ can promote positive adaptations in squat kinematics owing to its capacity to induce a more upright trunk position. Equivalent results have also been verified in resistance-trained practitioners who performed back squat with wooden blocks of $25 \mathrm{~mm}$ [16] or weightlifting shoes $[12,13]$. This adaptation leads to smaller overload in the lumbar region [24] and may potentially improve the technique in novices. By squatting with less anterior trunk inclination, novices are closer to a movement pattern that is observed in experienced practitioners [17]. Indeed, the maintenance of natural lumbar lordosis curvature, achieved by an upright trunk position, is related to lifting higher loads during the back squat, which suggests another positive outcome of squatting with elevated heels. Practical applications of these results may also be found in rehabilitation programs, whereas professionals can utilize this strategy to aid patients with movement restrictions in these joints, reducing load-related stress in the lumbar region and developing specific protocols to reach individualized goals [4].

Some previous research did not reveal adaptations in trunk inclination as described earlier by elevating the heels $[14,15]$. We believe, however, that methodological differences between our and their investigations can account for the divergent results. For instance, Lee et al. [14] analysed women, implemented a load of $80 \%$ of one-repetition maximum, and did not instruct participants to squat as deep as possible; in turn, Whitting et al. [15] also analysed loaded conditions (50\%, $70 \%$, and $90 \%$ of one-repetition maximum). Considering that there are significant differences in kinematics between sexes [25] and that loading affects movement kinematics [15] during back squat, we propose that these differences might explain the distinct outcomes between our analyses. More pressingly, the instruction not to squat as deep as possible hinders further comparisons between the experiments, especially when considering the notion of achieving maximal RoMs. It could be speculated that reduced anterior trunk inclination promoted by a heel elevation would only be observable when squatting at a full depth, as half-squats do not require excessive inclination of the trunk to maintain balance and perform the exercise.

Greater knee RoM values during a back squat with elevated heels, on the other hand, seem to be a more consistent finding in the literature [12-14, 16], especially when participants are asked to perform maximal RoM. This result should be highlighted, as larger RoM increases time under tension, which is a key variable in muscle hypertrophy protocols [26]. The increased RoM leads to superior stretching of the muscle fibres recruited for the movement in question, which induces greater muscle activation patterns in resistance training exercise [27]. Thus, larger RoMs enhance 2 important aspects related to strength and hypertrophy training: time under tension and muscle activation. These adaptations, therefore, are critical to practitioners seeking strength and muscle mass gains [28]. In line with this evidence, it has been verified that squatting at a greater depth induces additional hypertrophy in the gluteus maximus and adductor muscles when compared with half squats (knee joint stopping at $90^{\circ}$ of flexion) [3]. Thus, it is safe to suggest that using a wedge, as implied in our research, may be a promising approach to allow a greater depth while back squatting.

Regarding the ankle joint, significant differences were determined for dorsiflexion between W25 and W50, with increased RoM in the W50 condition. This result can be explained by the induced plantar flexion caused by the wooden block, which altered the joint kinematics, increasing its RoM. Furthermore, it is essential to mention that the lack of ankle mobility can be a factor contributing to an increase in anterior trunk inclination [29]. Hence, elevating the heel with a wooden block or iron plates could be an alternative to momentarily compensate impaired ankle mobility, inducing a more upright trunk position during back squat execution. With reference to the hip joint, our results were similar to the findings by Legg et al. [12], in which experienced practitioners did not present RoM alterations in this joint with or without the elevated heel. However, the present experiment is the first to report results of the hip joint in the frontal plane. Adduction and abduction RoM were not different between the wedges and barefoot conditions, which suggests that modifications in the heel height impact primarily on the flexion and extension movements in the sagittal plane.

To the best of our knowledge, this is the first experiment to analyse a 50-mm heel height while back squatting, showing an additional benefit compared 
with the 25-mm elevation. Even though previous analyses are divergent regarding the possible benefits of squatting with weightlifting shoes or a wooden block beneath the heels, most evidence points toward positive adaptions. We must also highlight that our participants had an average of 5-year experience with this exercise. Considering that trained subjects are less sensitive to adaptations because of their higher training levels, elevating the heel during back squat seems a promising strategy to improve movement kinematics. We should also consider that the back squat is used in many training programs, like protocols for rehabilitation [4], elderly functionality [30], and lower limb hypertrophy [2]. Therefore, our results can be applied to a variety of training programs. Our study, however, is not without limitations. The results are restricted to the current study design. It is uncertain whether elevating the heel during back squat would promote similar adaptations in other populations (i.e., women, novices, or older adults) or loading conditions.

\section{Conclusions}

We conclude that using a $25-$ or $50-\mathrm{mm}$ wooden block beneath the heel while back squatting is a viable alternative to weightlifting shoes, leading to a reduced trunk inclination and increased knee RoM in trained males. Hence, a wooden block might be an accessible and low-cost alternative to promote positive adaptations during back squat in a variety of training facilities and programs.

\section{Acknowledgements}

The authors would like to thank the participants who volunteered in this study and Bruno Giovanini for the artwork of Figure 1.

\section{Disclosure statement}

No author has any financial interest or received any financial benefit from this research.

\section{Conflict of interest}

The authors state no conflict of interest.

\section{References}

1. Schoenfeld BJ. Squatting kinematics and kinetics and their application to exercise performance. J Strength Cond Res. 2010;24(12):3497-3506; doi: 10.1519/JSC. 0b013e3181bac2d7.

2. Rossi FE, Schoenfeld BJ, Ocetnik S, Young J, Vigotsky A, Contreras B, et al. Strength, body composition, and functional outcomes in the squat versus leg press exercises. J Sports Med Phys Fitness. 2018;58(3):263270; doi: 10.23736/S0022-4707.16.06698-6.

3. Kubo K, Ikebukuro T, Yata H. Effects of squat training with different depths on lower limb muscle volumes. Eur J Appl Physiol. 2019;119(9):1933-1942; doi: 10.1007/ s00421-019-04181-y.

4. Everhart JS, Cole D, Sojka JH, Higgins JD, Magnussen RA, Schmitt LC, et al. Treatment options for patellar tendinopathy: a systematic review. Arthroscopy. 2017;33(4):861-872; doi: 10.1016/j.arthro.2016.11.007.

5. Watson SL, Weeks BK, Weis LJ, Harding AT, Horan SA, Beck BR. High-intensity resistance and impact training improves bone mineral density and physical function in postmenopausal women with osteopenia and osteoporosis: the LIFTMOR randomized controlled trial. J Bone Miner Res. 2018;33(2):211-220; doi: 10.1002/ jbmr.3284.

6. Caterisano A, Moss RF, Pellinger TK, Woodruff K, Lewis VC, Booth W, et al. The effect of back squat depth on the EMG activity of 4 superficial hip and thigh muscles. J Strength Cond Res. 2002;16(3):428-432; doi: 10.1519/ 1533-4287(2002)016<0428:TEOBSD>2.0.CO;2.

7. Wilk M, Golas A, Stastny P, Nawrocka M, Krzysztofik M, Zajac A. Does tempo of resistance exercise impact training volume? J Hum Kinet. 2018;62:241-250; doi: 10.2478/hukin-2018-0034.

8. Durall CJ, Manske R. Avoiding lumbar spine injury during resistance training. Strength Cond J. 2005;27(4): 64-72; doi: 10.1519/00126548-200508000-00011.

9. Yavuz HU, Erdag D. Kinematic and electromyographic activity changes during back squat with submaximal and maximal loading. Appl Bionics Biomech. 2017; 2017:9084725; doi: 10.1155/2017/9084725.

10. Raske $\AA$, Norlin R. Injury incidence and prevalence among elite weight and power lifters. Am J Sports Med. 2002;30(2):248-256; doi: 10.1177/03635465020300 021701.

11. Sinclair J, McCarthy D, Bentley I, Hurst HT, Atkins S. The influence of different footwear on 3-D kinematics and muscle activation during the barbell back squat in males. Eur J Sport Sci. 2015;15(7):583-590; doi: 10.1080/17461391.2014.965752.

12. Legg HS, Glaister M, Cleather DJ, Goodwin JE. The effect of weightlifting shoes on the kinetics and kinematics of the back squat. J Sports Sci. 2017;35(5):508515; doi: 10.1080/02640414.2016.1175652.

13. Sato K, Fortenbaugh D, Hydock DS. Kinematic changes using weightlifting shoes on barbell back squat. J Strength Cond Res. 2012;26(1):28-33; doi: 10.1519/ JSC.0b013e318218dd64.

14. Lee S-P, Gillis CB, Ibarra JJ, Oldroyd DF, Zane RS. Heel-raised foot posture does not affect trunk and lower extremity biomechanics during a barbell back squat in recreational weight lifters. J Strength Cond Res. 2019;33(3):606-614; doi: 10.1519/JSC.00000000000 01938. 
15. Whitting JW, Meir RA, Crowley-McHattan ZJ, Holding RC. Influence of footwear type on barbell back squat using 50, 70, and $90 \%$ of one repetition maximum: a biomechanical analysis. J Strength Cond Res. 2016; 30(4):1085-1092; doi: 10.1519/JSC.0000000000001 180.

16. Charlton JM, Hammond CA, Cochrane CK, Hatfield GL, Hunt MA. The effects of a heel wedge on hip, pelvis and trunk biomechanics during squatting in resistance trained individuals. J Strength Cond Res. 2017;31(6): 1678-1687; doi: 10.1519/JSC.0000000000001655.

17. Sayers MGL, Bachem C, Schütz P, Taylor WR, List R, Lorenzetti S, et al. The effect of elevating the heels on spinal kinematics and kinetics during the back squat in trained and novice weight trainers. J Sports Sci. 2020;38(9):1000-1008; doi: 10.1080/02640414.2020. 1738675 .

18. Krzyszkowski J, Kipp K. Load-dependent mechanical demands of the lower extremity during the back and front squat. J Sports Sci. 2020;38(17):2005-2012; doi: 10.1080/02640414.2020.1766738.

19. Faul F, Erdfelder E, Lang A-G, Buchner A. G*Power 3: a flexible statistical power analysis program for the social, behavioral, and biomedical sciences. Behav Res Methods. 2007;39(2):175-191; doi: 10.3758/bf03193146.

20. Winter M, Bunge C-A, Setti D, Petermann K. A statistical treatment of cross-polarization modulation in DWDM systems. J Light Technol. 2009;27(17):37393751; doi: 10.1109/JLT.2009.2025394.

21. Morris SB, DeShon RP. Combining effect size estimates in meta-analysis with repeated measures and independent-groups designs. Psychol Methods. 2002;7(1): 105-125; doi: 10.1037/1082-989x.7.1.105.

22. Cohen J. Statistical power analysis for the behavioral sciences. New York: Lawrence Erlbaum Associates; 1988.

23. Rhea MR. Determining the magnitude of treatment effects in strength training research through the use of the effect size. J Strength Cond Res. 2004;18(4):918920; doi: 10.1519/14403.1.

24. Butler RJ, Plisky PJ, Southers C, Scoma C, Kiesel KB. Biomechanical analysis of the different classifications of the Functional Movement Screen deep squat test. Sports Biomech. 2010;9(4):270-279; doi: 10.1080/14 763141.2010 .539623$.

25. McKean MR, Dunn PK, Burkett BJ. The lumbar and sacrum movement pattern during the back squat exercise. J Strength Cond Res. 2010;24(10):2731-2741; doi: 10.1519/JSC.0b013e3181e2e166.

26. Schoenfeld BJ, Ogborn DI, Krieger JW. Effect of repetition duration during resistance training on muscle hypertrophy: a systematic review and meta-analysis. Sports Med. 2015;45(4):577-585; doi: 10.1007/s40279015-0304-0.

27. Marcori A, Moura T, Okazaki V. Gastrocnemius muscle activation during plantar flexion with different feet positioning in physically active young men. Isokinet
Exerc Sci. 2017;25(2):121-125; doi: 10.3233/IES160654.

28. Schoenfeld BJ. The mechanisms of muscle hypertrophy and their application to resistance training. J Strength Cond Res. 2010;24(10):2857-2872; doi: 10.1519/ JSC.0b013e3181e840f3.

29. Fuglsang EI, Telling AS, Sørensen H. Effect of ankle mobility and segment ratios on trunk lean in the barbell back squat. J Strength Cond Res. 2017;31(11):30243033; doi: 10.1519/JSC.0000000000001872.

30. Edholm P, Strandberg E, Kadi F. Lower limb explosive strength capacity in elderly women: effects of resistance training and healthy diet. J Appl Physiol. 2017;123(1): 190-196; doi: 10.1152/japplphysiol.00924.2016. 